\title{
Conhecimento de adolescentes e jovens sobre questões relacionadas ao sexo, em uma escola pública de Monte Alegre do Piauí-PI
}

\section{Knowledge of adolescents and young people on issues related to sex, in a public school in Monte Alegre do Piaú-PI}

\author{
Patrícia Soares Pereira de Andrade (orcid.org/0000-0003-0017-6447)'1 \\ Karina Rodrigues de Sousa Barros (orcid.org/0000-0003-0936-6829)2 \\ Jéssica Pereira dos Santos (orcid.org/0000-0002-1639-517X)3 \\ Elaine Ferreira do Nascimento (orcid.org/0000-0002-1632-9148) ${ }^{4}$ \\ Polyanna Araújo Alves Bacelar (orcid.org/0000-0002-6509-2327) ${ }^{5}$
}

\begin{abstract}
Resumo
O presente estudo objetivou avaliar o conhecimento de adolescentes e jovens sobre questões relacionadas ao sexo, na cidade de Monte Alegre do Piauí (PI). Para isso, utilizou-se o método descritivo quantitativo por meio de questionário semiestruturado aplicado a 81 estudantes, com idade entre 16 a 23 anos, da escola Ginásio Estadual Senador Chagas Rodrigues. A coleta de dados evidenciou que 97,5\% ( $\mathrm{n}=79)$ dos participantes já haviam praticado sexo, tendo iniciado com idade média de 15,5 anos. O preservativo foi o método contraceptivo mais conhecido, no entanto, observou-se que os adolescentes e jovens não se preveniram em todas as relações sexuais, mesmo cientes das consequências da não prevenção. Percebeu-se fragilidade dialógica entre os indivíduos, família e escola; a conversação sobre o assunto no âmbito familiar e escolar ainda era receosa e pouco atrativa. Como conclusão deste estudo, sugere-se como necessário a implementação eficaz de políticas públicas que envolvam de forma educativa a escola, os jovens, seus familiares e amigos.
\end{abstract}

Palavras-chave: Desinformação. Educação sexual. Sexo inseguro.

\footnotetext{
1 Universidade Aberta do Brasil, Gilbués, Brasil. E-mail: patricia.c5@hotmail.com.

2 Universidade Aberta do Brasil, Gilbués, Brasil. E-mail: ka_rb_02@hotmail.com.

${ }^{3}$ Escritório Regional da Fiocruz Piauí, Teresina, Brasil. E-mail: jessik_ssantos@hotmail.com..

${ }^{4}$ Escritório Regional da Fiocruz Piauí, Teresina, Brasil. E-mail: negraelaine@gmail.com..

${ }^{5}$ Escritório Regional da Fiocruz Piauí, Teresina, Brasil. E-mail: polyannabio_gen@hotmail.com.
} 


\begin{abstract}
This present study aimed to assess the knowledge of adolescents and young people on issues related to sex, in the city of Monte Alegre do Piauí (PI). For this, the quantitative descriptive method was used through a semi-structured questionnaire applied to 81 students, aged between 16 and 23 years old, from the school Ginásio Estadual Senador Chagas Rodrigues. The data collection showed that $97.5 \%(n=79)$ of the participants had already had sex, having started with an average age of 15,5 years. The condom was the most known contraceptive method; however, it was observed that adolescents and young people did not prevent themselves in all sexual intercourse, even though they were aware of the consequences of non-prevention. Dialogical fragility was noticed among individuals, family and school; the conversation on the subject in the family and school environment was still fearful and unattractive. As a conclusion of this study, it is suggested as necessary the effective implementation of public policies that involve the school, young people, their families and friends in an educational way.
\end{abstract}

Keywords: Disinformation. Sexual education. Unsafe sex.

A Organização Mundial da Saúde (OMS) define adolescência e juventude a faixa etária compreendida entre 10 e 24 anos. Tal parâmetro é adotado no Brasil, que possui esse grupo como 19\% da população geral, equivalente a 34 milhões de indivíduos (Instituto Brasileiro de Geografia e Estatística [IBGE], 2010). É na adolescência que ocorrem profundas mudanças biopsicossociais, caracterizadas pelas modificações biológicas da puberdade, como crescimento rápido, surgimento das características sexuais secundárias e início da capacidade reprodutiva; além da conscientização da sexualidade, estruturação da personalidade, adaptação ambiental e integração social (Silva et al., 2015). É importante destacar que a puberdade não é caracterizada como sinônimo de adolescência, mas como uma parte dela (Lourenço \& Queiroz, 2010).

A adolescência é, geralmente, a fase em que a atividade sexual se inicia. No Brasil, durante a década de 80 , a média da idade para a realização da primeira relação em homens era de 15,6 anos e em mulheres 16,9 anos. Na década de 90, essa média variou para 15,3 e 16, respectivamente (Brasil, 1999). A partir do ano 2000, essas idades foram menores, como demonstrado na pesquisa de Tronco e Dell'Aglio (2012) sobre comportamento sexual de adolescentes, em que a média foi de 13,9 anos para homens e 15,5 para mulheres. Outros estudos encontraram idades também menores, correspondendo ao intervalo entre 14,8 a 15,23 anos, sem diferença significativa entre homens e mulheres (Bergamim \& Borges, 2009; Borges \& Schor, 2005; Silva et al., 2015). De forma geral, nota-se que os homens iniciam a 
vida sexual mais cedo, observação também evidenciada em estudos no México e na China (Barragán, Berenzon, Tiburcio, Bustos, \& Villatoro, 2019; Ma et al., 2009), enquanto as mulheres, nos últimos anos, tendem a se aproximar da média masculina.

Alguns fatores podem estar associados à iniciação sexual nessa faixa etária, como a maior permanência dos adolescentes e jovens em casa sem supervisão e ocupação (Cohen, Farley, Taylor, Martin, \& Schuster, 2002); falta de orientação na escola e ausência de diálogo na família sobre sexualidade (Lara \& Abdo, 2015); uso de álcool e substâncias ilícitas (Tilahun \& Ayele, 2013); abuso sexual na infância (Farid, Che' Rus, Dahlui, \& Al-Sadat, 2013) e; exposição a cenas eróticas (O’Hara, Gibbons, Gerrard, Li, \& Sargent, 2012). As implicações disso são o maior risco de relações desprotegidas e o comprometimento do processo natural de crescimento e desenvolvimento por consequência da gravidez não planejada e das Infecções Sexualmente Transmissíveis (IST) (Sanchez et al., 2013).

Em alguns países, a gravidez nesse grupo populacional vem sendo considerada problema de saúde pública, uma vez que pode acarretar transtornos físicos (como complicações obstétricas para a mãe e o recém-nascido) e impactos emocionais, sociais e econômicos que repercutem negativamente na consolidação dos projetos de vida do adolescente; problemas que refletem no aumento dos casos de abandono escolar (Pariz, Mengarda, \& Frizz, 2012; Taborda, Silva, Ulbricht, \& Nevez, 2014).

No Brasil, no ano 2000, foram identificados 0,9\% de nascidos vivos de mães entre 10 e 14 anos e 22,4\% entre 15 e 19 anos de idade (Filho, Sigrist, Souza, Mateus, \& Rassam, 2011). Esse cenário foi demonstrado na cidade de São Paulo, com 200 adolescentes grávidas, das quais 18,5\% $(\mathrm{n}=37)$ se encontravam na faixa etária entre 13 e 15 anos (Vieira, Bousquat, Barros, \& Alves, 2017). Já Hydall, Duarte e Costa (2018), ao estudarem 158 jovens de 10 a 19 anos que tiveram partos prematuros em Rio Branco (Acre), apontaram que, na faixa etária entre 15 a 19 anos, ocorreu um número expressivo de partos, correspondendo a 92\% ( $n=145)$. No Piauí, também foi revelado perfil semelhante em um estudo realizado em Teresina, com 464 mulheres que foram gestantes na adolescência, o qual apontou que $53,7 \%(n=294)$ realizaram a primeira relação sexual próxima à idade da menarca. O estudo apontou ainda que houve abandono escolar e despreparo da família e escola para orientação sobre o assunto (Nery, Mendonça, Gomes, Fernandes, \& Oliveira, 2011). 
Outro aspecto importante é o aumento do número de diagnósticos das IST entre adolescentes e jovens, que geralmente se disseminam por meio das primeiras experiências sexuais, facilitado pela desinformação (Castro, Caldas, Morcillo, Pereira, \& Velho, 2016). Essa situação evidencia o quanto é necessário investir continuamente em ações de caráter educativo que visem a redução dos fatores de riscos e possam contribuir para a cidadania (Bottega et al., 2016). Inevitavelmente, tal conjuntura remete à ineficácia de políticas públicas direcionadas não só a atender as demandas sexuais e reprodutivas da população jovem, vulneráveis aos riscos oriundos da relação sexual desprotegida, como também atender sua família, escola e comunidade, haja vista que a elaboração e implementação de tais políticas poderiam reduzir os indicadores de gravidez e das IST entre os jovens (Vieira et al., 2017).

Nesse sentido, para que o início da atividade sexual seja mais seguro, é preciso que o aprendizado da sexualidade não se limite apenas à genitalidade e ao coito. É pertinente que o início do uso de métodos contraceptivos seja estimulado antes do primeiro contato íntimo. Faz-se necessário conhecer melhor os pensamentos e tabus no que diz respeito à sexualidade dos(as) jovens e adolescentes, para que seja possível abordá-los de maneira satisfatória, contribuindo de forma efetiva para o desenvolvimento e crescimento saudável.

Diante do exposto, esta pesquisa teve por objetivo avaliar o conhecimento de adolescentes e jovens sobre questões relacionadas ao sexo, na cidade de Monte Alegre do Piauí (PI). O intuito foi de caracterizar o perfil sociodemográfico e identificar os conhecimentos sobre métodos contraceptivos, IST e gravidez nesses períodos do desenvolvimento humano.

\section{Métodos e delineamento do estudo}

Trata-se de um estudo descritivo, com abordagem quantitativa, que foi realizado em uma escola da rede pública denominada Ginásio Estadual Senador Chagas Rodrigues, no município de Monte Alegre do Piauí (PI), nos meses de março a maio de 2018. Estavam devidamente matriculados na escola 95 alunos distribuídos na IV $\left(6^{\circ}\right.$ e $7^{\circ}$ anos do ensino fundamental), $V\left(8^{\circ}\right.$ e $9^{\circ}$ anos do ensino fundamental), $V I\left(1^{\circ}\right.$ e $2^{\circ}$ anos do ensino médio) e 
VII ( $3^{\circ}$ ano do ensino médio) etapas da Educação de Jovens e Adultos - EJA. Desses, 81 compuseram a amostra do estudo, com faixa etária entre 16 e 23 anos de idade e de ambos os sexos.

Foram considerados como critérios de inclusão idade entre 16 e 23 anos e aceite como participante da pesquisa através da assinatura do Termo de Consentimento/Assentimento Livre e Esclarecido. As informações foram coletadas por meio de um questionário semiestruturado baseado em pesquisa bibliográfica, contendo 19 questões que abordaram as seguintes variáveis: sexo, idade, estado civil, escolaridade, área de moradia, se já praticou relação sexual e com qual idade iniciou, aspectos sobre gravidez, conhecimentos sobre os métodos contraceptivos e IST. Os dados coletados foram tabelados em planilha eletrônica no programa Microsoft Excel 2010 e analisados através de distribuição de frequências. Em sequência, as respostas das frequências absolutas foram calculadas, transformadas em porcentagem e dispostas em tabelas e gráficos para uma melhor compreensão das informações.

Esta pesquisa recebeu aprovação do Comitê de Ética em Pesquisa com Seres Humanos da Faculdade de Ciências e Tecnologia do Maranhão - FACEMA, sob o número do CAAE 84487918.6.0000.8007, conforme Resolução 510/2016 do Conselho Nacional de Saúde (Brasil, 2016).

\section{Resultados e discussão}

Dos 81 alunos que participaram deste estudo, 75,3\% eram do ensino médio $(n=61)$ e $56,8 \%$ pertencentes ao sexo feminino $(n=46)$. A maioria $(59,3 \%, n=48)$ dos entrevistados residia na área urbana, dos quais $79 \%(n=64)$ eram solteiros, $13,6 \%(n=11)$ em união estável e $7,4 \%(n=6)$ casados (Tabela1). 


\section{Tabela 1}

Perfil sociodemográfico dos adolescentes e jovens entrevistados no Ginásio Estadual Senador Chagas Rodrigues em Monte Alegre do Piauí (PI), Brasil

\begin{tabular}{lcc}
\hline Variáveis & N & Frequência Relativa (\%) \\
\hline Estado civil & 06 & 7,4 \\
Casado & 64 & 79,0 \\
Solteiro & 11 & 13,6 \\
União estável & 35 & 43,2 \\
\hline Sexo do adolescente & 46 & 56,8 \\
Masculino & & \\
Feminino & 33 & 40,7 \\
\hline Mora em que zona & 48 & 59,3 \\
Rural & & 24,7 \\
Urbana & 20 & 75,3 \\
\hline Nível de escolaridade & 61 & \\
Ensino Fundamental & & \\
Ensino Médio & & \\
\hline
\end{tabular}

Fonte: Elaborada pelos autores.

Em tempo, o aspecto de gênero remete a uma reflexão no contexto histórico de alfabetização no Brasil, com grupos etários do sexo feminino apresentando um aumento gradativo nesse índice, atenuando a caracterização secular de desigualdade de gênero, em que mulheres são tratadas como inferiores aos homens (Ferreira, Nascimento, Pedrosa, \& Monte, 2017). A taxa contemporânea de alfabetização do sexo feminino tem se apresentado um pouco superior em comparação ao masculino. Além disso, o índice de mulheres estudando na EJA, no período de 2004 a 2013 , passou de $88,8 \%$ para $91,8 \%$, aumento proporcional ao dos homens, que subiu de 88,3\% para 91,3\% (Brasil, 2015a). De maneira geral, é possível observar que os adolescentes e jovens têm considerado a educação formal como via de acesso para a conquista do trabalho, e, por consequência, da estabilidade financeira (Marcelino, Catão, \& Lima, 2009). Contudo, a exclusão escolar feminina ainda se 
mantém acentuada (Corcetti, Souza, \& Loreto, 2018; IBGE \& Conselho Nacional de Educação, 1961).

Em relação à atividade sexual, quando os adolescentes e jovens foram questionados se já praticaram sexo, quase todos, $97,5 \%(n=79)$, responderam que sim, relatando vida sexual ativa. O público investigado também foi indagado sobre qual idade ocorreu o primeiro intercurso sexual e a média foi de 15,5 anos. Destaca-se que houve relatos de iniciação com $9(1,2 \%, n=1), 10(1,2 \%, n=1)$ e $12(1,2 \%, n=1)$ anos de idade, todos masculinos (Figura 1). A prática sexual antes dos 13 anos também foi relatada no Rio Grande do Sul e em outras regiões, como em Cabo Verdo e no Canadá (Gonçalves et al., 2015; Phillips, King, Michaelson, \& Pickett, 2019; Tavares, Kanikadan, Alencar, \& Schor, 2011). Isso expõe o fato de que, mesmo antes do pleno desenvolvimento do aparelho reprodutor, a criança já vivencia a sexualidade, visto que esta não depende da maturação biológica (Lima, Oliveira, Souza, Pereira, \& Sant'Anna, 2010). Contudo, é um período no qual não possui condições biopsicossociais adequadas para conduzir os diversos aspectos relacionados ao sexo e à sua saúde (Gonçalves et al., 2015).

\section{Figura 1}

Idade do início de relação sexual dos adolescentes entrevistados no Ginásio Estadual Senador Chagas Rodrigues em Monte Alegre do Piauí (PI), Brasil

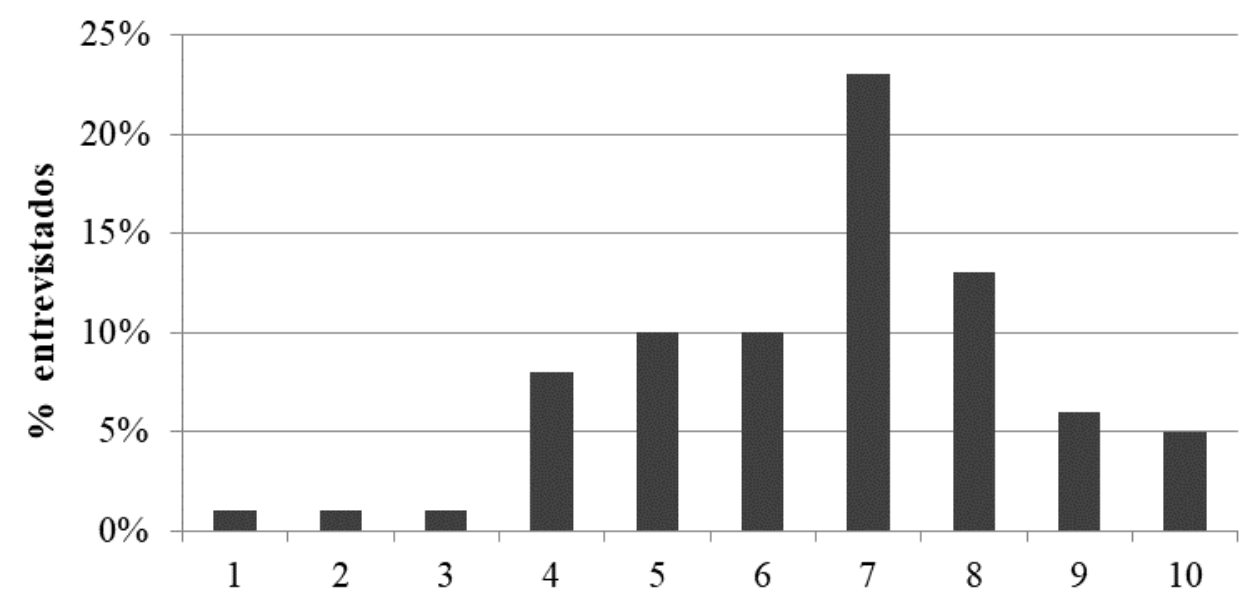

Fonte: Elaborada pelos autores. 
Mais de um quinto dos adolescentes no Brasil, entre 12 a 17 anos de idade, já iniciaram a prática sexual (Ramiro et al., 2015), e a quantidade dos que tiveram relação íntima antes dos 15 anos é maior entre os meninos (Oliveira-Campos et al., 2014). A prática sexual na adolescência pode estar relacionada à exigência de grupos, incentivos dos meios de comunicação, além da ausência de diálogo com familiares (Amoras, Campos, \& Beserra, 2015; Spinola, Béria, \& Schermann, 2017). Essa prática se configura como um comportamento de risco, tendo em vista que o adolescente exposto a essas situações pode ter um aumento no número de parceiros sexuais durante a vida e, consequentemente, maior chance de exposição a alguma IST e de gravidez não planejada (Cunha, Saraiva, \& Barros, 2017; Silva et al., 2015).

Em todo o mundo, mais de dez milhões de infectados pelo Vírus da Imunodeficiência Humana (HIV) estão situados na faixa etária entre 15 e 24 anos de idade. No Brasil, entre os anos de 2005 e 2015, o número de casos entre pessoas nessa faixa etária duplicou, demonstrando a relevância epidemiológica desse segmento populacional. Ainda é importante destacar que, de 1980 a 2015, foram notificados 89.112 casos de Síndrome da Imunodeficiência Humana (AIDS) no Brasil entre pessoas na faixa etária de 10 a 24 anos, correspondendo a 8,9\% do total de notificação no país. No estado do Piauí, de 1986 a 2015, foram notificados 6.004 casos, o que corresponde a $5,1 \%$ das notificações totais da região Nordeste (Brasil, 2015b). Diante disso, o planejamento e a realização de ações de educação dirigidas a esses adolescentes, no âmbito da saúde sexual e reprodutiva, são de fundamental importância (Brasil, 2007).

Dentre os métodos contraceptivos conhecidos entre os adolescentes e jovens deste estudo, o preservativo foi o mais citado, correspondendo a $76,5 \%(n=62)$, seguido da pílula anticoncepcional com 22,2\% $(n=18)$. Apenas 1,2\% $(n=1)$ não soube opinar, indicando que o público investigado apresentou conhecimento sobre os métodos, porém, uma minoria ainda não os reconhece, tornando-se ainda mais vulneráveis. $O$ atual método contraceptivo mais utilizado é o preservativo masculino $(88,9 \%, n=71)$, seguido pelo feminino $(3,7 \%, n=3)$, anticoncepcional oral $(3,7 \%, n=3)$ e pela pílula do dia seguinte $(2,4 \%, n=2)$ (Figura 2). 


\section{Figura 2}

Atual método contraceptivo usado entre os adolescentes e jovens entrevistados no Ginásio Estadual Senador Chagas Rodrigues em Monte Alegre do Piauí (PI), Brasil

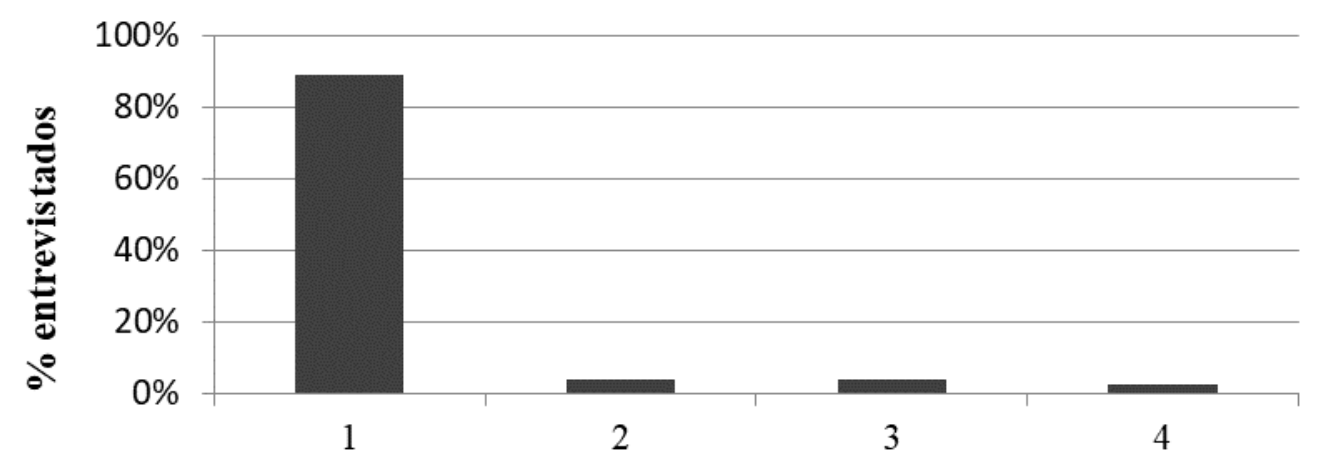

Fonte: Elaborada pelos autores.

Em um estudo realizado por Oliveira, Maximino, Silva e Silva (2015), o preservativo foi citado $(48,7 \%, 14 / 29)$ como método preventivo mais conhecido e usado nas relações sexuais, seguido pelo anticoncepcional oral com 20,6\% (6/29), entre os adolescentes de uma escola municipal do estado da Paraíba. Em outra pesquisa desenvolvida por Moreira, Pereira, Brito, Duarte e Barros (2012) com 53 adolescentes de Pernambuco sobre IST e métodos contraceptivos, também ficou evidenciado que $66 \%$ (35/53) consideram o preservativo como a forma mais adequada para a prevenção dessas infecções e da gravidez, não descartando os outros meios, como o uso da pílula anticoncepcional $(28,3 \%, 15 / 53)$.

Quanto ao uso do preservativo em todas as relações sexuais, constatou-se que uma proporção de $53,1 \%(n=43)$ dos adolescentes e jovens não o usam. Os preservativos masculinos e femininos são barreiras contra a transmissão das IST, além de protegerem contra o risco de uma concepção indesejada (Soares, Silva, Silva, Freire, \& Nogueira, 2017). Santos et al. (2016), em um estudo sobre a adesão ao uso do preservativo masculino por adolescentes escolares, evidenciaram que os fatores que justificaram a não utilização desse método preventivo foram a não portabilidade do mesmo no momento do ato sexual $(20,3 \%)$, a diminuição do prazer nas relações (19\%) e a confiança no parceiro $(6,3 \%)$.

Alguns fatores podem ter contribuído para a não utilização de preservativos pelo público investigado no presente estudo, seja o modelo feminino, pela dificuldade no 
manuseio, de acesso ou pela indisponibilidade; seja o masculino, devido à iniciativa do uso ser geralmente proveniente do parceiro (Raiford, Seth, Braxton, \& DiClemente, 2013). Outras circunstâncias também estão associadas ao não uso de preservativos, como o baixo nível socioeconômico e cultural e as diferenças de gênero, considerando as concepções e preocupações vivenciadas nos relacionamentos afetivo-sexuais (Andrade, Silva, Silva, Oliveira, \& Montenegro, 2012).

Em um estudo realizado com adolescentes em três capitais brasileiras, foi evidenciado que o uso de preservativos diminui significativamente na última relação sexual quando comparada à primeira, particularmente entre as mulheres. Destas, pouco mais de um terço teve apenas um parceiro sexual, ao passo que as trajetórias masculinas foram caracterizadas por uma maior variedade de parceiras, implicando no maior uso do preservativo. A mudança de status do relacionamento também implica na diminuição do uso de preservativo (Teixeira, Knauth, Fachel, \& Leal, 2006).

Além disso, a atividade sexual desprotegida pode trazer grandes repercussões negativas na saúde dos adolescentes, podendo causar uma gravidez de risco bem como o desenvolvimento do câncer relacionado à infecção pelo Papiloma Vírus Humano (HPV) (Elias et al., 2017; Instituto Nacional do Câncer, 2015). A vulnerabilidade dessa população também está relacionada às questões socioeconômicas, como a baixa escolaridade, o escasso poder aquisitivo, a não utilização de preservativos e o consumo de drogas (Brasil, 2015b; Oliveira, Abud, Inagaki, Alves, \& Matos, 2018).

Quanto ao questionamento a respeito da busca de informações sobre temas relacionados ao sexo, observou-se que $33,3 \%(n=27)$ dos entrevistados relataram obtê-las através de diálogo com os pais, 19,8\% $(n=16)$ através da internet, 17,3\% $(n=14)$ com amigos (as), 14,8\% ( $n=12)$ na escola, 8,6\% ( $n=7)$ com irmãos e 6,2\% $(n=5)$ em outros locais (Figura 3). 


\section{Figura 3}

Questionamento a respeito da fonte de informações sobre sexo dos adolescentes e jovens entrevistados no Ginásio Estadual Senador Chagas Rodrigues em Monte Alegre do Piauí (PI), Brasil

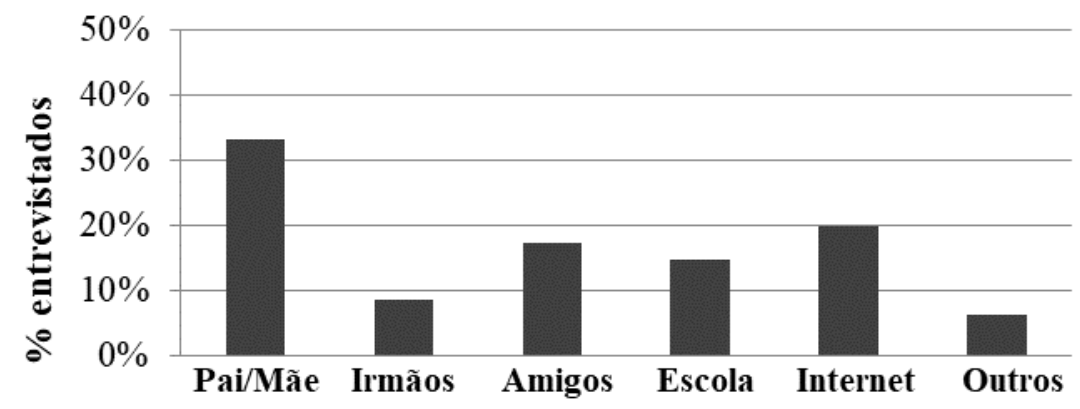

Fonte: Elaborada pelos autores.

A comunicação acerca de assuntos relacionados ao sexo é limitada no âmbito familiar $(n=34)$ quando comparada à quantidade de adolescentes que buscam informações através da internet, amigos, na escola ou em outros locais $(n=47)$; no entanto, é maior quando comparada apenas à variável escola $(n=7)$. Essa fragilidade dialógica entre escola e família pode prejudicar o conhecimento efetivo sobre essa temática, estimulando a procura tardia pelos serviços de saúde e induzindo os adolescentes e jovens a se informarem de forma incorreta ou equivocada (Fontes et al., 2017).

É importante salientar que os meios de comunicação contribuem no sentido de orientar e divulgar aspectos relacionados à educação sexual, no entanto, podem distorcer fatos e informações, criando dúvidas ainda maiores (Lima et al., 2010). A limitação familiar quanto a discussão sobre o tema em questão geralmente ocorre porque os pais e/ou responsáveis consideram possível que o diálogo incentive a prática, ou ainda, porque atribuem valor negativo ao assunto, por não terem recebido educação sexual em seu passado (Gonçalves, Faleiro, \& Malafaia, 2013).

Além disso, é provável que ocorra sentimento de vergonha e o despreparo na abordagem sobre o assunto, configurando-se um tabu (Nery et al., 2015). Esses aspectos devem ser trabalhados para que a família seja fonte segura de conhecimento sobre o assunto e para que seja proporcionado um ambiente de confiança. Isso favorece o 
encorajamento do adolescente e do jovem pela busca de orientações que podem contribuir para a redução de problemas pessoais e sociais (Genz, Meincke, Carret, Corrêa, \& Alves, 2017). O acesso à educação e à informação sobre essa temática é considerado indispensável na prevenção dos comportamentos sexuais de risco (World Health Organization [WHO], 2010).

Em contrapartida, a maioria dos adolescentes passa parte do tempo na escola, que é o ambiente adequado para a aprendizagem e deveria ser a opção de espaço fundamental para a educação sexual, ao oferecer diálogo em que se possam discutir suas dúvidas e desenvolver programas relacionados à saúde (Aspy et al., 2012). Dessa forma, a escola proporcionaria maiores conhecimentos em relação aos comportamentos sexuais, o que seria considerado uma atitude preventiva (Ramiro, Reis, Matos, \& Diniz, 2011).

Quanto ao conhecimento dos adolescentes sobre as IST, ficou evidenciado que a AIDS é a mais conhecida, com $95 \%$ das respostas, seguida da sífilis, com 3,7\%, e 1,2\% não souberam responder. Uma pesquisa similar realizada com adolescentes no estado de Minas Gerais apontou AIDS, sífilis e gonorreia como as IST mais relatadas (Elias et al., 2017). Em outro estudo realizado por Carvalho, Pinto e Santos (2018), foi identificado que os adolescentes conheciam dez IST, estando a AIDS como a mais citada. O maior conhecimento relacionado à AIDS provavelmente ocorreu devido às divulgações publicitárias a partir da década de 1980, e à ênfase na gravidade da doença (Portinari \& Wolfgang, 2017).

Interrogou-se ainda sobre a principal via de transmissão das IST e as respostas foram: sexo sem preservativo $(96,3 \%)$, contato com a roupa íntima $(2,4 \%)$ e o contato com feridas $(1,2 \%$. Diante desses resultados, observou-se que os adolescentes, de modo geral, demonstraram conhecimentos sobre as formas de contágio e a necessidade do uso do preservativo como meio de proteção, o que não é suficiente para incentivar a adoção de atitudes efetivas na prevenção. Segundo o estudo de Cunha, Oliveira, Oliveira e Praxedes (2016) com 241 adolescentes da cidade de Goiânia, 94,2\% reconhecem a via sexual como forma de contágio das IST. Entretanto, 20,3\% também citaram o uso de talheres, banheiro e um aperto de mão. Os mitos relacionados às vias de transmissão das IST precisam ser desconstruídos, tendo em vista que essas informações se disseminam facilmente. 
Dentre as opiniões em relação às maiores mudanças que ocorrem na vida das adolescentes diante de uma gravidez, foram relatadas: as dificuldades financeiras e emocionais (34,5\%), a evasão escolar (24,6\%), a dificuldade de emprego $(11,1 \%)$ e a perda da liberdade social $(11,1 \%)$; uma minoria, $8,6 \%$, relatou não identificar nenhuma mudança (Figura 4).

\section{Figura 4}

Mudanças que ocorrem na vida das adolescentes diante de uma gravidez pelos adolescentes entrevistados no Ginásio Estadual Senador Chagas Rodrigues em Monte Alegre do Piauí (PI), Brasil

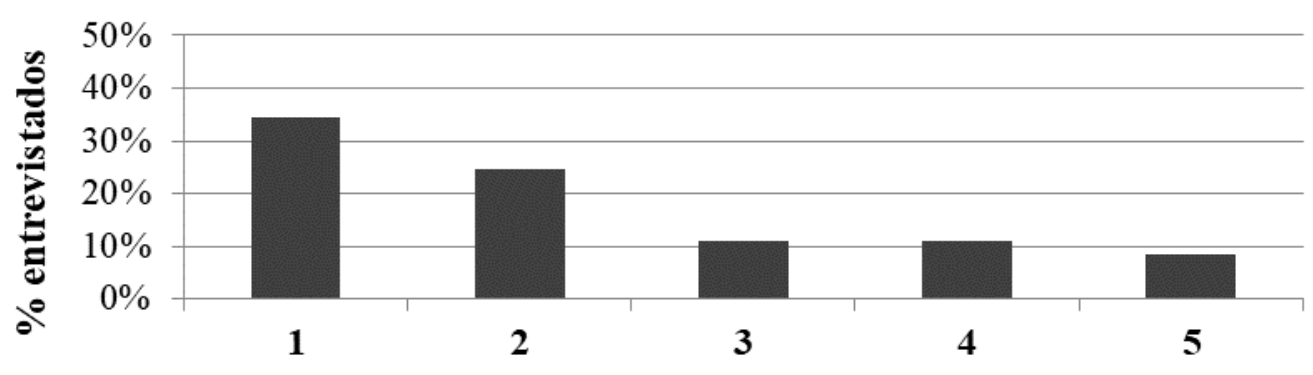

Fonte: Elaborada pelos autores.

Diversos fatores relacionados à gravidez na adolescência são considerados pela OMS como de alto risco pela susceptibilidade a problemas de crescimento e desenvolvimento emocionais, comportamentais, educacionais e de aprendizado, bem como as complicações na gestação e trabalho de parto (Mendes, 2017). Além desses fatores, há uma preocupação com a descontinuidade dos estudos e formação profissional, cujas consequências são o abandono da escola e o despreparo para o mercado de trabalho (Pontes, Sousa, Oliveira, Pedreira, \& Costa, 2012; Rocha, Souza, \& Bittar, 2017). 


\section{Considerações finais}

A pesquisa evidenciou o levantamento do perfil dos adolescentes e jovens, identificando que a maioria era do sexo feminino, solteira, do ensino médio e morava na zona urbana. Quanto à atividade sexual, quase todos já a vivenciaram, tendo iniciado com a idade média de 15,5 anos, idade em que a relação sexual pode ser caracterizada como um comportamento de risco. A busca de informações sobre temas relacionados ao sexo no âmbito familiar ocorreu de forma limitada, quando comparada ao conjunto das outras fontes de acesso à informação, e em maior proporção quando comparada apenas à escola.

Tal fato pode estar associado à deficiência de discussões no âmbito familiar sobre sexualidade, sendo de extrema importância que os pais desmistifiquem seus tabus e assumam seu papel frente à educação de seus filhos. A escola e a implementação de políticas públicas, por sua vez, precisam buscar estratégias que favoreçam o debate sobre essa temática em uma ação conjunta com a participação dos pais, educadores e profissionais da saúde, contribuindo assim para a atenção integral à saúde do adolescente.

O método contraceptivo que os adolescentes e jovens mais conheciam foi o preservativo, porém, não se preveniam em todas as relações sexuais, demonstrando que conhecimento sobre os métodos contraceptivos nem sempre se converte em relações sexuais seguras.

Ainda, foi possível identificar que o público estudado possui noções sobre IST, sendo as mais citadas a AIDS e a sífilis. Além disso, consideraram o não uso do preservativo como o principal mecanismo de contágio dessas infecções, o que não é suficiente para sensibilizálos ao seu uso habitual. Quanto à gravidez não planejada, as limitações financeiras e emocionais, a evasão escolar e a dificuldade de emprego foram as consequências mais relatadas.

É de suma importância que ocorra promoção em saúde para os adolescentes e jovens, com informações e debates por meio de atividades escolares que associem famílias, profissionais da saúde, todo o corpo gestor e docentes das escolas. Essa dinâmica deve proporcionar a autonomia necessária para as decisões de uma relação sexual mais segura, promovendo um espaço de discussão dessa temática, com metodologias educativas sobre 
sexualidade e saúde reprodutiva. Portanto, é necessário que os adolescentes e jovens tenham plenos conhecimentos sobre todos os aspectos que envolvam a sexualidade e o sexo, para que assim se mantenham prevenidos de quaisquer riscos à saúde.

Uma limitação do presente estudo foi a reduzida expressão dos adolescentes e jovens, provavelmente por constrangimento em abordar questões relacionadas ao sexo. Apesar dessa limitação, houve adesão em torno de $85 \%$ dos estudantes matriculados na escola. Vale ressaltar a importância deste estudo por se tratar da primeira investigação sobre a temática no município, o que poderá servir como base para outras pesquisas de natureza transversal e longitudinal.

\section{Referências}

Amoras, B. C., Campos, A. R., \& Beserra, E. P. (2015). Reflexões sobre vulnerabilidade dos adolescentes a infecções sexualmente transmissíveis. Revista Eletrônica de Humanidades do Curso de Ciências Sociais da UNIFAP, 8(1), 163-171. https://periodicos.unifap.br/index.php/pracs/article/view/1668/

Andrade, S. S. C., Silva, F. M. C., Silva, M. S. S., Oliveira, S. H. S., \& Montenegro, S. M. S. L. (2012). Prevenção de vulvovaginite: utilizando as falas das mulheres para elaboração de orientações em saúde. Revista de Enfermagem UFPE On Line, 6(2), 339-345. https://doi.org/10.5205/reuol.2052-14823-1-LE.0602201212

Aspy, C. B., Vesely, S. K., Oman, R. F., Tolma, E., Rodine, S., Marshall, L. et al. (2012). School-related assets and youth risk behaviors: Alcohol consumption and sexual activity. Journal of school health, 82(1), 3-10. https://doi.org/10.1111/j.1746$1561.2011 .00661 . x$

Barragán, V., Berenzon, S., Tiburcio, M., Bustos, M., \& Villatoro, J. (2019). Factors associated with sexual debut in mexican adolescents: results of the national survey on drug use among students in 2014. The Journal of Sexual Medicine, 16(3), 418-426. https://doi.org/10.1016/j.jsxm.2019.01.008 
Bergamim, M. D., \& Borges, A. L. V. (2009). Fatores associados à iniciação sexual entre adolescentes da zona oeste do município de São Paulo. Revista Gaúcha de Enfermagem, 30(3), 420-428. https://seer.ufrgs.br/RevistaGauchadeEnfermagem/article/view/9316

Borges, A. L. V., \& Schor, N. (2005). Início da vida sexual na adolescência e relações de gênero: um estudo transversal em São Paulo, Brasil, 2002. Cadernos de Saúde Pública, 21, 499-507. https://doi.org/10.1590/S0102-311X2005000200016

Bottega, A., Canestrini, T., Rodrigues, M. A., Rampelotto, R. F., Santos, S. O., Silva, D. C. et al. (2016). Abordagem das Doenças Sexualmente Transmissíveis na adolescência: revisão de literatura. Saúde (Santa Maria), 91-104. https://doi.org/10.5902/2236583421481

Brasil, Conselho Nacional de Saúde. Ministério da Saúde. (2016). Resolução 510, de 07 de Abril de 2016. Normas aplicáveis a pesquisas em Ciências Humanas e Sociais. Brasília, DF: Ministério da Saúde. http://bvsms.saude.gov.br/bvs/saudelegis/cns/2016/res0510_07_04_2016.html

Brasil, República Federativa do Brasil. Ministério da Educação. (2015a). Plano Nacional de Educação PNE 2014-2024: Linha de Base. Instituto Nacional de Estudos e Pesquisas Educacionais Anísio Teixeira (Inep). Brasília, DF: Ministério da Educação. http:/ / portal.inep.gov.br/documents / 186968 / 485745/Plano+Nacional+de+Educa\%C3\% A7\%C3\%A3o+PNE+2014-2024++Linha+de+Base/c2dd0faa-7227-40ee-a520$12 \mathrm{c} 6 \mathrm{fc} 77700 \mathrm{f}$ ?version $=1.1$

Brasil, República Federativa do Brasil. Ministério da Saúde. (1999). Comportamento Sexual da População Brasileira e Percepções sobre o HIV/AIDS. Relatório Final de Pesquisa. Ministério da Saúde - SPS - CNDST/HIV/AIDS. Brasília, DF: Ministério da Saúde. https://www.nepo.unicamp.br/publicacoes/relatorios/aids.pdf

Brasil, República Federativa do Brasil. Ministério da Saúde. (2007). Secretaria de Atenção à Saúde. Saúde integral de adolescentes e jovens: orientações para a organização de serviços de saúde. (2005). Brasília, DF: Ministério da Saúde. http://bvsms.saude.gov.br/bvs/publicacoes/saude_adolescentes_jovens.pdf

Brasil, República Federativa do Brasil. Ministério da Saúde. Secretaria de Vigilância em Saúde, Departamento de DST, Aids e Hepatites Virais. (2015b). Boletim epidemiológico: Aids e 
DST. Brasília, DF: Ministério da Saúde. http://www.aids.gov.br/system/tdf/pub/2015/90/boletim_hepatites_2015.pdf?file $=1 \& t$ ype $=$ node $\&$ id $=90 \&$ force $=1$

Carvalho, G. R. O., Pinto, R. G. S., \& Santos, M. S. (2018). Conhecimento sobre as infecções sexualmente transmissíveis por estudantes adolescentes de escolas públicas. $\begin{array}{lllll}\text { Adolescência } & \& & \text { Saúde, } & \text { 7-17. }\end{array}$ http://www.adolescenciaesaude.com/detalhe_artigo.asp?id=703\#

Castro, E. L. D., Caldas, T. A. D., Morcillo, A. M., Pereira, E. M. D. A., \& Velho, P. E. N. F. (2016). O conhecimento e o ensino sobre doenças sexualmente transmissíveis entre universitários. Ciência \& Saúde Coletiva, 27(6), 1975-1984. https:// doi.org/10.1590/1413-81232015216.00492015

Cohen, D. A., Farley, T. A., Taylor, S. N., Martin, D. H., \& Schuster, M. A. (2002). When and where do youths have sex? The potential role of adult supervision. Pediatrics, $110(6)$, e66-e66.

Corcetti, E., Souza, S. P., \& Loreto, M. D. S. (2018). O "Programa Mulheres Mil" no Espírito Santo: uma política pública educacional de equidade de gênero? Ensaio: Avaliação $e$ Políticas Públicas em Educação, 26(100), 1-33. https://doi.org/10.1590/s010440362018002601027

Cunha, M. P., Oliveira, B. F. R., Oliveira, I. C. M., \& Praxedes, L. K. S. (2016). Análise do conhecimento sobre DSTS/AIDS entre adolescentes em Goiânia, Goiás. Revista da $\begin{array}{llll}\text { Universidade } \quad \text { Vale } & \text { Rio } & \text { 650-658. }\end{array}$ https:// doi.org/10.5892/ruvrd.v14i2.2856

Cunha, N. C., Saraiva, I. S., \& Barros, M. D. M. (2017). Pesquisa sobre sexualidade e gravidez na adolescência: uma reflexão acerca da importância da articulação do conhecimento através da participação ativa dos estudantes. Vittalle-Revista de Ciências da Saúde, 29(2), 11-22. https://doi.org/10.14295/vittalle.v29i2.7029

Elias, T. C., Santos, T. N., Soares, M. B. O., Gomes, N. S., Parreira, B. D. M., \& Silva, S. R. (2017). Conhecimento de alunas de uma universidade federal sobre doenças sexualmente transmissíveis [Female federal university's students' knowledge of sexually 
transmitted diseases]. Revista Enfermagem UERJ, 25, e10841. https:// doi.org/10.12957/reuerj.2017.10841

Farid, N. N., Che'Rus, S., Dahlui, M., \& Al-Sadat, N. (2013). Determinants of sexual intercourse initiation among incarcerated adolescents: a mixed-method study. Singapore Medical Journal, 54(12), 695-701. https://doi.org/10.11622/smedj.2013244

Ferreira, B. O., Nascimento, E. F., Pedrosa, A. I. S., \& Monte, L. M. I. (2017). Vivências de travestis no acesso ao SUS. Physis Revista de Saúde Coletiva, 27(4), 1023-1038. https://doi.org/10.1590/S0103-73312017000400009

Filho, F. P., Sigrist, R. M. S., Lobianco, L., Mateus, D. C., \& Rassam, E. (2011). Perfil epidemiológico da grávida adolescente no município de Jundiaí e sua evolução em trinta anos. Adolescência $\quad e \quad$ Saúde, 8(1), 21-27. https://cdn.publisher.gn 1 .link/adolescenciaesaude.com/pdf/v8n 1 a04.pdf

Fontes, M. B., Crivelaro, R. C., Scartezini, A. M., Lima, D. D., Garcia, A. D. A., \& Fujioka, R. T. (2017). Fatores determinantes de conhecimentos, atitudes e práticas em DST/Aids e hepatites virais, entre jovens de 18 a 29 anos, no Brasil. Ciência \& Saúde Coletiva, 22(4), 1343-1352. https://doi.org/1 0.1590/1413-81232017224.12852015

Genz, N., Meincke, S. M. K., Carret, M. L. V., Corrêa, A. C. L., \& Alves, C. N. (2017). Doenças Sexualmente Transmissíveis: conhecimento e comportamento sexual de adolescentes. Texto $\quad \& \quad$ Contexto Enfermagem, 26(2), $1-12$. https://doi.org/10.1590/0104-07072017005100015

Gonçalves, H., Machado, E. C., Soares, A. L. G., Camargo-Figuera, F. A., Seerig, L. M., Mesenburg, M. A. et al. (2015). Início da vida sexual entre adolescentes (10 a 14 anos) e comportamentos em saúde. Revista Brasileira de Epidemiologia, 18(1), 25-41. https://doi.org/10.1590/1980-5497201500010003

Gonçalves, R. C., Faleiro, J. H., \& Malafaia, G. (2013). Educação sexual no contexto familiar e escolar: impasses e desafios. Holos, 5, 251-263. https://doi.org/10.15628/holos.2013.784 
Hydall, A. R. S., Duarte, R. N., \& da Costa, R. S. L. (2018). Partos prematuros em adolescentes em Rio Branco-Acre no ano de 2015. DêCiência em Foco, 2(1), 34-44. http://revistas.uninorteac.com.br/index.php/DeCienciaemFoco0/article/view/144 Instituto Brasileiro de Geografia e Estatística. (2010). Censo demográfico 2010. Rio de $\begin{array}{lll}\text { Janeiro: } & \text { IBGE. } & \text { https://www.ibge.gov.br/estatisticas- }\end{array}$ novoportal/sociais / populacao/9662-censo-demografico-2010.html?=\&t=destaques

Instituto Brasileiro de Geografia e Estatística \& Conselho Nacional de Estatística. (1961). Contribuições para o estudo da demografia no Brasil. In A. P. Figueiredo (Ed.), $A$ alfabetização da população do Brasil, segundo o sexo, a idade e a cor (pp. 392-400). Rio de Janeiro: IBGE/CNE.

Instituto Nacional do Câncer. (2015). Estimativa 2016: incidência de câncer no Brasil. Rio de Janeiro: INCA. $\quad$ http://santacasadermatoazulay.com.br/wpcontent/uploads/2017/06/estimativa-2016-v1 1.pdf

Lara, L. A. D. S., \& Abdo, C. H. N. (2015). Aspectos da atividade sexual precoce. Revista Brasileira de Ginecologia e Obstetrícia, 37(5), $\quad$ 199-202. https:// doi.org/10.1590/SO100-720320150005207

Lima, A. A., Oliveira, D. C., Souza, E. S., Pereira, J. A., \& Sant'Anna, V. L. L. (2010). Educação sexual infantil: A interação entre a família e escola como fator determinante para uma educação eficaz. $\quad$ Pedagogia em Ação, 2(1), 83-88. http://periodicos.pucminas.br/index.php/pedagogiacao/article/view/4484

Lourenço, B., \& Queiroz, L. B. (2010). Crescimento e desenvolvimento puberal na adolescência. Revista de Medicina, 89(2), 70-75. https://doi.org/10.11606/issn.16799836.v89i2p70-75

Ma, Q., Ono-Kihara, M., Cong, L., Xu, G., Pan, X., Zamani, S. et al. (2009). Early initiation of sexual activity: a risk factor for sexually transmitted diseases, HIV infection, and unwanted pregnancy among university students in China. BMC Public Health, 9(1), 111. https:// doi.org/10.1186/1471-2458-9-111

Marcelino, M. Q. D. S., Catão, M. D. F. F. M., \& Lima, C. M. P. D. (2009). Representações sociais do projeto de vida entre adolescentes no ensino médio. Psicologia: ciência $e$ profissão, 29(3), 544-557. https://doi.org/10.1590/S1414-98932009000300009 
Mendes, A. M. (2017). Plano de ação para redução dos índices de gravidez na adolescência entre os jovens atendidos pela Estratégia Saúde da Família 01 do Município de Marcolândia-Piauí [Trabalho de Conclusão de Curso, Universidade Federal do Maranhão]. Acervo UNA SUS. https://ares.unasus.gov.br/acervo/handle/ARES/8067

Moreira, S. B., Pereira, P. S., Brito, A. M., Duarte, A. E., \& Barros, L. M. (2012). DSTs: percepção dos estudantes da Escola São Vicente de Paula, Exu-PE. Enciclopédia Biosfera, Centro Científico $\quad$ Conhecer, $\quad 8(15), \quad 2078-2088$. https://www.conhecer.org.br/enciclop/2012b/ciencias\%20humanas/dsts.pdf

Nery, I. S., Gomes, K. R. O., Barros, I. C., Gomes, I. S., Fernandes, A. C. N., \& Viana, L. M. M. (2015). Fatores associados à reincidência de gravidez após gestação na adolescência no Piauí, Brasil. Epidemiologia e Serviços de Saúde, 24(4), 671-680. https://doi.org/10.5123/S1679-49742015000400009

Nery, I. S., Mendonça, R. C. M., Gomes, I. S., Fernandes, A. C. N., \& Oliveira, D. C. (2011). Reincidência da gravidez em adolescentes de Teresina, PI, Brasil. Revista Brasileira de Enfermagem, 64(1), 31-37. https://doi.org/10.1590/S0034-71672011000100005

O'Hara, R. E., Gibbons, F. X., Gerrard, M., Li, Z., \& Sargent, J. D. (2012). Greater exposure to sexual content in popular movies predicts earlier sexual debut and increased sexual risk taking. Psychological science, 23(9), 984-993. https:// doi.org/10.1177/0956797611435529

Oliveira, P. S., Abud, A. C. F., Inagaki, A. D. M., Alves, J. A. B., \& Matos, K. F. (2018). Vulnerabilidade de adolescentes às doenças sexualmente transmissíveis na atenção primária. Revista de Enfermagem UFPE, 12(3), 53-62. https://doi.org/10.5205/19818963-v12i3a24120p753-762-2018

Oliveira, R. N., Maximino, D. A. F. M., Silva, P. E., \& Silva, V. C. L. (2015). Iniciação sexual de adolescentes e conhecimento dos métodos contraceptivos. Ciência da Saúde Nova Esperança, 13(2), 66-76. $\quad$ http://www.facene.com.br/wpcontent/uploads/2010/11/Inicia----o-sexual_PRONTO.pdf

Oliveira-Campos, M., Nunes, M. L., Madeira, F. C., Santos, M. G., Bregmann, S. R., Malta, D. C. et al. (2014). Comportamento sexual em adolescentes brasileiros, Pesquisa Nacional 
de Saúde do Escolar (PeNSE 2012). Revista Brasileira de Epidemiologia, 17(suppl 1), 116 30. https://doi.org/10.1590/1809-4503201400050010

Pariz, J., Mengarda, C. F., \& Frizzo, G. B. (2012). A atenção e o cuidado à gravidez na adolescência nos âmbitos familiar, político e na sociedade: uma revisão da literatura. Saúde e Sociedade, 21(3), 623-636. https://doi.org/10.1590/S010412902012000300009

Phillips, S. P., King, N., Michaelson, V., \& Pickett, W. (2018). Sex, drugs, risk and resilience: analysis of data from the Canadian Health Behaviour in School-aged Children (HBSC) study. European journal of public health, 29(1), 38-43. https://doi.org/10.1093/eurpub/cky169

Pontes, L. C., Sousa, W. S., Oliveira, D. C., Pedreira, I. Q., \& Costa, S. M. (2012). As implicações da gravidez na adolescência: uma revisão bibliográfica. Revista Interdisciplinar NOVAFAPI, 5(1), 55-60. https://revistainterdisciplinar.uninovafapi.edu.br/revistainterdisciplinar/v5n1/rev/rev1_ v5n1.pdf

Portinari, D. B., \& Wolfgang, S. M. B. M. (2017). Imagens e marcas: um imaginário ligado à epidemia de HIV-Aids no Brasil. A/ceu, 17(34), 45-60. http://revistaalceu.com.pucrio.br/media/alceu34_pp45-60.pdf

Raiford, J. L., Seth, P., Braxton, N. D., \& DiClemente, R. J. (2013). Masculinity, condom use self-efficacy and abusive responses to condom negotiation: the case for HIV prevention for heterosexual African-American men. Sexual health, 10(5), 467-469. https://doi.org/10.1071/SH13011

Ramiro, L., Reis, M., Matos, M. G., \& Diniz, J. A. (2011). Sex education among Portuguese adolescent students. Procedia-Social and Behavioral Sciences, 29, 493-502. https:// doi.org/10.1016/j.sbspro.2011.11.198

Ramiro, L., Windlin, B., Reis, M., Gabhainn, S. N., Jovic, S., Matos, M. G. et al. (2015). Gendered trends in early and very early sex and condom use in 20 European countries from 2002 to 2010. The European Journal of Public Health, 25(suppl 2), 65-68. https://doi.org/10.1093/eurpub/ckv030 
Rocha, R. M. N., Souza, P. C., \& Bittar, C. M. L. (2017). Relatos sobre a percepção da gravidez para um grupo de adolescentes e jovens mulheres. Gerais: Revista Interinstitucional de Psicologia, 1O(1), 59-68. http://pepsic.bvsalud.org/pdf/gerais/v10n1/07.pdf

Sanchez, Z. M., Nappo, S. A., Cruz, J. I., Carlini, E. A., Carlini, C. M., \& Martins, S. S. (2013). Sexual behavior among high school students in Brazil: alcohol consumption and legal and illegal drug use associated with unprotected sex. Clinics, 68(4), 489-494. http://doi.org/10.6061/clinics/2013(04)09

Santos, C. P., Barboza, E. C. S., Freitas, N. O., Almeida, J. C., Dias, A. C., \& Araújo, E. C. (2016). Adesão ao uso do preservativo masculino por adolescentes escolares. Revista Brasileira de Pesquisa em Saúde/Brazilian Journal of Health Research, 18(2), 60-70. https://doi.org/10.21722/rbps.v18i2.15085

Silva, A. D. S. N., Silva, B. L. C. N., Júnior, S., Silva, M. C. F. D., Guerreiro, J. F., \& Sousa, A. D. S. C. D. (2015). Início da vida sexual em adolescentes escolares: um estudo transversal sobre comportamento sexual de risco em Abaetetuba, Estado do Pará, Brasil. Revista Pan-Amazônica de Saúde, 6(3), 27-34. https://doi.org/10.5123/S217662232015000300004

Soares, J. P., Silva, A. C. O., Silva, D. M., Freire, M. E. M., \& Nogueira, J. A. (2017). Prevalência e fatores de risco para o HIV/AIDS em populações vulneráveis: uma revisão integrativa de literatura. Arquivos Catarinenses de Medicina, 46(4), 182-194. http://www.acm.org.br/acm/seer/index.php/arquivos/article/view/126

Spinola, M. C. R., Béria, J. U., \& Schermann, L. B. (2017). Fatores associados à iniciação sexual em mães de 14 a 16 anos em Porto Alegre/RS, Brasil. Ciência \& Saúde Coletiva, 22(11), 3755-3762. https:// doi.org/10.1590/1413-812320172211.00082016

Taborda, J. A., Silva, F. D., Ulbricht, L., \& Neves, E. B. (2014). Consequências da gravidez na adolescência para as meninas considerando-se as diferenças socioeconômicas entre elas. Cadernos Saúde Coletiva, 22(1), 16-24. https://doi.org/10.1590/1414$462 \times 201400010004$

Tavares, C. M., Kanikadan, P. Y. S., Alencar, A. P., \& Schor, N. (2011). Beginning of sexual life of adolescents in Santiago Island, Cape Verde, West Africa. Journal of Human Growth and Development, 2 1(3), 771-779. https://doi.org/10.7322/jhgd.20029 
Teixeira, A. M. F. B., Knauth, D. R., Fachel, J. M. G., \& Leal, A. F. (2006). Adolescentes e uso de preservativos: as escolhas dos jovens de três capitais brasileiras na iniciação e na última relação sexual. Cadernos de Saúde Pública, 22(7), 1385-1396. https:// doi.org/10.1590/S0102-311X2006000700004

Tilahun, M., \& Ayele, G. (2013). Factors associated with age at first sexual initiation among youths in Gamo Gofa, south west Ethiopia: a cross sectional study. BMC Public Health, 13(1), 622. https://doi.org/10.1186/1471-2458-13-622

Tronco, C. B., \& Dell'Aglio, D. D. (2012). Caracterização do comportamento sexual de adolescentes: iniciação sexual e gênero. Gerais: Revista Interinstitucional de Psicologia, 5(2), 254-269. http:// pepsic.bvsalud.org/pdf/gerais/v5n2/v5n2a06.pdf

Vieira, E. M., Bousquat, A., Barros, C. R. S., \& Alves, M. C. G. P. (2017). Gravidez na adolescência e transição para a vida adulta em jovens usuárias do SUS. Revista de Saúde Pública, 51(25), 1-11.https://doi.org/10.1590/S1518-8787.2017051006528

World Health Organization. (2010). WHO Regional Office for Europe and BZgA-Standards for sexuality education in Europe: A framework for policy makers, educational and health authorities and specialists. Cologne: Federal Centre for Health Education.

Recebido em: 23/7/2018

Aprovado em: 12/4/2019 